Service social

\title{
Le soutien social des personnes âgées au carrefour des services de l'État
}

\section{Michèle Clément et Jacques Roy}

Volume 41, numéro 1, 1992

L'avenir des services ou services d'avenir

URI : https://id.erudit.org/iderudit/706557ar

DOI : https://doi.org/10.7202/706557ar

Aller au sommaire du numéro

\section{Éditeur(s)}

École de service social de l'Université Laval

ISSN

1708-1734 (numérique)

Découvrir la revue

Citer cet article

Clément, M. \& Roy, J. (1992). Le soutien social des personnes âgées au carrefour des services de l'État. Service social, 41(1), 46-66.

https://doi.org/10.7202/706557ar
Résumé de l'article

Cet article présente les résultats d'une recherche menée auprès de personnes âgées dans la Municipalité régionale de comté de Pabok en Gaspésie. Les auteurs discutent de la prépondérance des facteurs tenant au soutien naturel (en particulier celui de la famille) et à l'environnement social dans l'orientation des personnes âgées vers les établissements de services publics. Ils se penchent en outre sur la nécessité de repenser l'articulation entre services formels et informels dans la perspective d'une plus grande complémentarité entre eux et d'un soutien accru de l'État aux aidants naturels. Des pistes d'intervention sont également suggérées; elles portent tout particulièrement sur le développement de services de soutien à domicile et de répit visant à soutenir les aidants naturels oeuvrant auprès de personnes âgées en perte d'autonomie. 
Michèle Clément, agente de recherche au Centre de recherche sur les services communautaires, Université Laval.

Jacques Roy, membre de l'équipe de recherche en gérontologie et attaché de recherche au Centre de recherche sur les services communautaires, Université Laval.

\title{
Le soutien social des personnes âgées au carrefour des services de l'État
}

\author{
Michèle Clément \\ Jacques Roy
}

La question du soutien social apporté aux personnes âgées vivant dans leur milieu naturel fait l'objet d'une attention privilégiée dans le secteur de la recherche sociale depuis une dizaine d'années. En particulier, nous faisons référence aux études qui ont cherché à éclairer les dynamiques existant entre aidants et aidés et les limites du fardeau des aidants naturels œuvrant auprès des personnes âgées en perte d'autonomie; parmi les études québécoises menées sur le sujet, signalons entre autres les travaux de Bolduc et Garant (1990), Corin (1984), Jutras et Veilleux (1989), Lepage (1989), Lesemann et Chaume (1989), Paquette (1988) et Stryckman et Paré-Morin (1987).

Cette quête d'une meilleure connaissance des réseaux de soutien social a été motivée, entre autres, par la volonté récente de l'État de partager, avec la famille et les ressources communautaires, les responsabilités de l'avenir quant à la prise en charge des personnes âgées (Jutras et Veilleux, 1989). La désinstitutionnalisation et la non-institutionnalisation de cette clientèle figurent à l'agenda gouvernemental bien que les limites du soutien social et les paramètres de partage entre l'État, la famille et les communautés soient encore très peu connus. À cet égard, soulignons que l'étude de Jutras et Renaud (1987), portant sur les personnes âgées et les aidants naturels, a mis en perspective l'inexistence relative de re- 
cherches ayant porté sur l'interaction entre l'aide naturelle apportée par l'entourage et la communauté d'une part et les services de l'État d'autre part. Depuis, au moins deux exceptions ont surgi : la recherche conduite par Lesemann et Chaume (1989) sur le maintien à domicile des personnes âgées en sérieuse perte d'autonomie et notre projet de recherche qui a porté sur la problématique des personnes âgées vivant à domicile dans la Municipalité régionale de comté de Pabok, sous l'angle de l'interface recherchée entre le soutien social et les services de l'État (Clément, 1990).

Les résultats de l'étude pouvant éclairer la relation entre le soutien social (famille, communauté...) et les services du réseau des affaires sociales font l'objet du présent article. En fait, nous nous limiterons aux constats qui alimentent la réflexion autour de cette relation car, en soi, l'objet de la recherche était de portée plus large, embrassant des pans variés de la réalité des personnes âgées.

\section{La recherche : contexte, objet et méthodologie}

L'idée de la recherche a surgi d'une table de concertation représentant différents établissements du réseau des affaires sociales dans la MRC de Pabok en Gaspésie. Celle-ci s'interrogeait en effet sur le phénomène grandissant du recours aux différents services du réseau par les personnes âgées vivant dans leur milieu naturel. On pense ici, en particulier, à l'utilisation des services de maintien à domicile et à l'hébergement institutionnel en centre d'accueil et en centre hospitalier de soins de longue durée. C'est dans le contexte de ce questionnement, enfin, que la problématique de la contribution du soutien social dans le milieu comme réponse potentielle aux besoins des personnes âgées et de sa capacité de réduire, sinon d'infléchir la tendance à utiliser les services du réseau des affaires sociales s'est posée. Ces premières intuitions demandaient à être vérifiées, et ce sont elles qui sont devenues les fils conducteurs de la recherche.

D'une façon plus précise, mentionnons que l'objet de la recherche consistait en l'étude des facteurs qui conditionnent l'orientation des aînés hommes et femmes vers les différents services du réseau public ainsi qu'en la compréhension des mécanismes conduisant à l'engorgement des services. De portée plus générale, l'objet de la recherche nous a donc conduits à explorer des dimensions tenant à la santé, aux caractéristiques sociales des personnes âgées et à celles de leur environnement; cependant, comme nous le verrons plus loin, la question du soutien social s'est 
avérée centrale dans l'examen de la problématique de notre recherche.

Sur le plan méthodologique, deux démarches distinctes mais interreliées ont été réalisées. Une première, de nature quantitative, a servi à construire le portrait général de la réalité des personnes âgées vivant à domicile sur le territoire de la MRC de Pabok et à dégager les principaux facteurs associés à l'utilisation qu'elles font des services du réseau de la santé. La seconde démarche (celle-là de type qualitatif et complémentaire à la première) a permis d'effectuer une plongée en approfondissant, cette fois, la dynamique des relations existant entre les personnes âgées, les aidants naturels principaux et l'ensemble de la communauté (amis, voisins, groupes sociaux, etc.).

La démarche quantitative s'est appuyée sur les résultats de questionnaires d'enquête distribués au printemps de 1989 à un échantillon représentatif de 289 personnes âgées de 65 ans et plus, vivant à domicile et résidant sur le territoire de la MRC de Pabok. L'échantillon a été tiré de façon aléatoire à partir des listes électorales provinciales de 1985. Comme il s'est écoulé un délai de quatre ans entre les deux (établissement des listes électorales et enquête auprès des personnes âgées), nous avons sélectionné un plus grand nombre de sujets afin d'avoir une liste de remplacement de noms étant donné les modifications (décès, déménagement, hébergement institutionnel...) ayant pu survenir pendant cette période. Une liste de remplacement automatique de noms a donc été utilisée, suivant en cela certains critères précis (âge, sexe, municipalité). Seuls les sujets ayant refusé de répondre au questionnaire (9\%) ont été retranchés de l'échantillon initial. Une double stratification (selon l'âge et la provenance géographique) a été appliquée sur l'échantillon, afin de tenir compte de l'importance relative du groupe des 65-74 ans et de celui des 75 ans et plus ainsi que de la localité de résidence au sein de la MRC. Sur le plan statistique, des analyses bivariées ont été effectuées en utilisant le $\mathrm{Chi}^{2}(\leqslant 0,05)$ comme mesure d'association et le coefficient Gamma $(\geqslant 0,40)$ pour apprécier la force des relations observées. Nous avons retenu un seuil minimal de 0,40 pour le coefficient Gamma en raison du fait que les mêmes variables étaient utilisées fréquemment. Le questionnaire d'enquête couvrait six dimensions de la réalité des personnes âgées : les caractéristiques démographiques et socio-économiques, les types de ménage, l'habitation, l'environnement social et physique, la santé et les services.

En ce qui a trait au volet qualitatif de la recherche, il a été réalisé à partir d'entrevues semi-dirigées auprès de 20 personnes âgées ayant répondu au questionnaire d'enquête et de 10 aidants naturels 
principaux. Des entrevues ont également été menées auprès d'intervenants mais, aux fins du présent article, nous n'en rendrons pas compte car le matériel obtenu ne porte pas spécifiquement sur notre sujet. Par ailleurs, dans le cas des personnes âgées sélectionnées (20), rappelons qu'il s'agit d'un groupe en perte d'autonomie présentant une ou plusieurs des caractéristiques suivantes : habiter seul, considérer la résidence comme un fardeau important, se percevoir en mauvaise santé, être affecté par des invalidités ou avoir réduit son rythme de vie, se considérer comme inactif, se sentir souvent déprimé ou triste et se sentir débordé par les responsabilités en vivant à domicile. Autre critère retenu : sauf exception, les personnes étaient âgées de 75 ans et plus.

Ces entrevues visaient principalement l'approfondissement de certaines dimensions déjà traitées dans le volet quantitatif de l'étude, en plus de la connaissance des stratégies développées par les personnes âgées pour faire face à leur perte d'autonomie. Par ce moyen, nous avons aussi tenté d'approfondir les facteurs liés au soutien social (famille, communauté...), d'y tracer les contours des dynamiques existantes. Ces informations obtenues à la fois auprès des personnes âgées et des aidants principaux ont été colligées et catégorisées à l'aide de grilles préalablement construites autour des grands thèmes retenus dans le plan de la recherche. Une analyse de contenu a été effectuée sur le matériel des entrevues.

Ces deux démarches (quantitative et qualitative) se sont articulées à partir d'un cadre théorique de nature écologique, inspiré des grandes catégories de Bronfenbrenner (1979), où le micro, le méso, l'exo et le macro-système forment les bases du modèle d'analyse. Notre hypothèse théorique était ainsi formulée : un déséquilibre dans l'environnement, qu'il s'exprime dans l'un ou l'autre des systèmes ou encore entre deux systèmes ou plus, va engendrer une consommation accrue des services socio-sanitaires afin de contrebalancer le déséquilibre observé. Afin d'appuyer l'examen de cette hypothèse, nous avons sélectionné des variables de type environnemental, telles que la nature et la fréquence des contacts avec le milieu, l'aide naturelle existante, la participation aux groupes communautaires, les conditions de transport et d'habitation, les loisirs, etc. Ces variables ont été mises en relation avec le recours aux services publics (principalement les services de maintien à domicile et d'hébergement institutionnel), afin de vérifier leur degré d'association avec ce recours. Parallèlement, le même exercice s'est effectué avec d'autres types de variables tenant principalement aux caractéristiques individuelles des personnes âgées et au champ de la santé. De cette façon, nous avons pu apprécier l'importance relative des différentes catégories de varia- 
bles associées au recours aux services et soupeser la valeur de notre hypothèse théorique initiale.

\section{Les résultats de la recherche}

Comme dans la section portant sur la méthodologie, nous distinguerons les résultats provenant de l'enquête par questionnaire (volet quantitatif) de ceux émergeant des entrevues individuelles semi-dirigées (volet qualitatif). Volontairement, nous nous en tiendrons aussi aux informations les plus utiles pour la compréhension de la problématique du soutien social en rapport avec celle des services du réseau des affaires sociales.

\section{Le volet quantitatif}

Le portrait général des personnes âgées nous révèle certains traits qui méritent d'être soulignés. Notre échantillon se compose essentiellement de personnes âgées vivant à domicile en milieu rural, où $78 \%$ sont propriétaires de leur résidence (ce qui est un trait distinctif des milieux ruraux en comparaison avec la ville où les proportions de propriétaires sont manifestement moins élevées); dans $38 \%$ des cas, elles résident avec un conjoint ou une conjointe et $19 \%$ vivent seules; le phénomène de cohabitation avec les enfants est significativement plus manifeste dans ce milieu qu'ailleurs au Québec (37\% des personnes âgées dans I'échantillon).

D'une façon générale, les personnes âgées se disent satisfaites des relations qu'elles entretiennent avec les personnes qui partagent le même toit qu'elles ( $96 \%$ ) et avec leur milieu en général $(97 \%)$. Il s'agit d'une première aperception de la relation personnes âgées-milieu naturel; d'autres informations viennent documenter cette relation. Ainsi, par exemple, l'étude nous apprend que plus de $90 \%$ des personnes âgées peuvent compter sur l'aide de quelqu'un (voisins, enfants, conjoints) en cas d'urgence, que les aînés ont des contacts réguliers avec leurs enfants ( 80 \% plus d'une fois par semaine), avec des membres de la parenté (63\% au moins une fois par semaine) ainsi qu'avec des amis et des voisins ( $68 \%$ au moins une fois par semaine). En tout état de cause, $83 \%$ d'entre eux reçoivent aussi des appels téléphoniques à une fréquence au moins hebdomadaire. Sur le plan de l'engagement communautaire, une bonne proportion de personnes âgées participeraient activement à des groupes sociaux de leur milieu ( $47 \%$ chez les $65-$ 74 ans, $39 \%$ chez les 75 ans et plus), tandis que $61 \%$ aideraient d'autres personnes; on note ici des traits témoignant d'une soli- 
darité intra et intergénérationnelle bien réelle. Toujours sous le même angle, les personnes âgées qui reçoivent de leur milieu naturel une aide leur permettant de réaliser des activités quotidiennes (32\% des personnes âgées) voient cette aide fournie principalement par le conjoint ou la conjointe $(25 \%)$ ou les enfants (24\%); suivent de loin les autres membres de la parenté (11\%), l'aide privée rémunérée $(10 \%)$, les amis et voisins $(7 \%)$ et les groupes bénévoles $(3 \%)$.

Voilà qui exprime les principales caractéristiques de la relation personne âgée-environnement, puisées dans le volet quantitatif de la recherche. À ce tableau sommaire, il faudrait ajouter que $27 \%$ des personnes âgées nous ont signalé rencontrer des difficultés et des contraintes importantes relativement au transport; dans un même ordre d'idée, il a été révélé que certains lieux publics sont difficilement accessibles pour le cinquième des personnes âgées de l'étude.

Pour explorer davantage notre hypothèse de départ, à savoir que c'est le déséquilibre dans l'environnement de la personne âgée qui engendre l'utilisation des services du réseau public, nous avons mis en relation dans une seconde étape l'ensemble des variables du questionnaire avec trois variables clés nous permettant de qualifier ce recours : 1) les personnes âgées recevant déjà des services à domicile du CLSC La Saline; 2) les personnes âgées ne recevant pas des services à domicile du CLSC, mais qui considèrent qu'elles devraient en recevoir étant donné leur état, enfin; 3) les personnes âgées désirant être hébergées en institution (centre d'accueil, centre hospitalier de longue durée...)

Les résultats de la mise en relation des variables sont présentés au tableau qui suit. Ils nous permettent de dégager quelles sont les caractéristiques de l'environnement des aînés les plus susceptibles d'être associées à un recours aux services institutionnels. Deux constats émergent : 1) la prépondérance d'éléments associés à l'absence de soutien du milieu naturel comme facteurs de risque positivement liés au recours à des services du réseau des affaires sociales et; 2) la faible influence qu'exerceraient des facteurs de type santé sur le recours aux services.

De fait, les résultats contenus dans le tableau nous fournissent une configuration à trois paliers de facteurs associés au recours aux services : un premier palier (celui offrant les associations les plus significatives avec les services) porte sur des dimensions reliées à l'absence de soutien du conjoint, d'enfants, d'amis ou de voisins auprès des personnes âgées, dimensions du reste communes aux trois variables clés retenues. Un second palier concerne l'environnement social et physique élargi de la personne âgée; sous cette 
rubrique, on regroupe des facteurs tels l'isolement social, l'absence de loisirs, les problèmes de transport, l'inactivité dans la communauté chez la personne âgée. Au regard des résultats enregistrés, ce second palier se situe au fond dans le prolongement immédiat du premier, tout en étant moins déterminant que celui-ci mais présentant des associations plus significatives que le troisième palier qui se compose des facteurs classiques de type santé, tels l'état de santé perçu, les invalidités, les hospitalisations... Les résultats de notre étude font donc ressortir le fait que la question

\section{TABLEAU 1}

\section{Profil indicatif des facteurs associés à l'orientation des personnes âgées vers les services à domicile et I'hébergement institutionnel'}

\begin{tabular}{|c|c|c|c|c|c|}
\hline \multicolumn{2}{|c|}{$\begin{array}{l}\text { Personnes âgées recevant } \\
\text { des services à domicile du } \\
\text { CLSC }\end{array}$} & \multicolumn{2}{|c|}{$\begin{array}{l}\text { Personnes âgées ne re- } \\
\text { cevant pas de services à } \\
\text { domicile du CLSC mais } \\
\text { considérant qu'elles } \\
\text { nécessitent des services à } \\
\text { domicile }\end{array}$} & \multicolumn{2}{|c|}{$\begin{array}{l}\text { Personnes âgées désirant } \\
\text { être hébergées en institu- } \\
\text { tion (CA ou } \mathrm{CH})\end{array}$} \\
\hline Facteurs & Gamma & Facteurs & $\mathrm{mma}$ & Facteurs & $\mathrm{mma}$ \\
\hline $\begin{array}{l}\text { Ne pouvant comp- } \\
\text { ter sur l'aide d'en- } \\
\text { fants en cas d'ur- } \\
\text { gence. } \\
\text { Chi non sign. }\end{array}$ & 0,73 & $\begin{array}{l}\text { Ne pouvant comp- } \\
\text { ter sur l'aide d'amis } \\
\text { ou de voisins en } \\
\text { cas d'urgence. } \\
\text { Chi }{ }^{2} \text { non sign. }\end{array}$ & & $\begin{array}{l}\text { Ne pouvant comp- } \\
\text { ter sur l'aide d'un } \\
\text { conjoint ou d'une } \\
\text { conjointe pour } \\
\text { l'entretien ménager. } \text { Chi }^{2} \text { non sign. }\end{array}$ & \\
\hline $\begin{array}{l}\text { Rythme de vie } \\
\text { réduit par maladie } \\
\text { ou blessure. } \\
\text { Chi }^{2} 0,003\end{array}$ & 0,63 & $\begin{array}{l}\text { Ne pouvant comp- } \\
\text { ter sur l'aide d'un } \\
\text { conjointe ou d'une } \\
\text { conjointe pour } \\
\text { l'entretien ménager. } \\
\text { Chi' } 0,015\end{array}$ & 0,53 & $\begin{array}{l}\text { Aucun loisir. } \\
\mathrm{Chi}^{2} 0,002\end{array}$ & 0,64 \\
\hline $\begin{array}{l}\text { Inactif ou inactive } \\
\text { chez soi. Chi } 0,001\end{array}$ & 0,59 & $\begin{array}{l}\text { Résidence consi- } \\
\text { dérée comme un } \\
\text { fardeau sur le plan } \\
\text { de l'entretien in- } \\
\text { térieur. Chi' } 0,000\end{array}$ & 0,51 & $\begin{array}{l}\text { Locataires, pen- } \\
\text { sionnaires. } \\
\mathrm{Chi}^{2} \text { non sign. }\end{array}$ & 0,59 \\
\hline
\end{tabular}




\begin{tabular}{|c|c|c|c|c|c|}
\hline \multicolumn{2}{|c|}{$\begin{array}{l}\text { Personnes âgées recevant } \\
\text { des services à̀ domicile du } \\
\text { CLSC }\end{array}$} & \multicolumn{2}{|c|}{$\begin{array}{l}\text { Personnes âgées ne re- } \\
\text { cevant pas de services à } \\
\text { domicile du CLSC mais } \\
\text { considérant qu'elles } \\
\text { nécessitent des services à } \\
\text { domicile }\end{array}$} & \multicolumn{2}{|c|}{$\begin{array}{l}\text { Personnes âgées désirant } \\
\text { être hébergées en institu- } \\
\text { tion (CA ou } \mathrm{CH})\end{array}$} \\
\hline Facteurs & Gamma & Facteurs & Camma & Facteurs & amma \\
\hline $\begin{array}{l}\text { Loisirs sans } \\
\text { activités physiques. } \\
\text { Chi }^{2} 0,052\end{array}$ & 0,54 & $\begin{array}{l}\text { Inactif ou inactive } \\
\text { chez soi. } \mathrm{Chi}^{2} 0,003\end{array}$ & 0,49 & $\begin{array}{l}\text { Pas le sentiment } \\
d^{\prime} \text { avoir sa place } \\
\text { parmi ses proches. } \\
\text { Chi }^{2} \text { non sign. }\end{array}$ & 0,59 \\
\hline $\begin{array}{l}\text { Souffre de solitude. } \\
\mathrm{Chi}^{2} 0,000\end{array}$ & 0,50 & $\begin{array}{l}\text { Apporte rarement } \\
\text { de l'aide aux } \\
\text { autres. } \text { Chi }^{2} 0,000\end{array}$ & 0,45 & $\begin{array}{l}\text { Ne pouvant comp- } \\
\text { ter sur l'aide d'amis } \\
\text { ou de voisins en } \\
\text { cas d'urgence. } \\
\text { Chi }^{2} \text { non sign. }\end{array}$ & 0,56 \\
\hline $\begin{array}{l}\text { Inactif ou inactive } \\
\text { dans la commu- } \\
\text { nauté. } \mathrm{Chi}^{2} 0,016\end{array}$ & 0,48 & $\begin{array}{l}\text { Problèmes } \\
\text { de transport. } \\
\text { Chi }^{2} 0,000\end{array}$ & 0,44 & $\begin{array}{l}\text { Loisirs sans ac- } \\
\text { tivités physiques. } \\
\text { Chi non sign. }\end{array}$ & 0,53 \\
\hline $\begin{array}{l}\text { Perception d'une } \\
\text { santé déficiente ou } \\
\text { mauvaise. } \\
\mathrm{Chi}^{2} 0,005\end{array}$ & 0,45 & $\begin{array}{l}\text { Rythme de vie } \\
\text { réduit par maladie } \\
\text { ou blessure. } \\
\text { Chi }^{2} 0,000\end{array}$ & 0,43 & $\begin{array}{l}\text { Services à domicile } \\
\text { jugés insuffisants. } \\
\mathrm{Chi}^{2} \text { non sign. }\end{array}$ & 0,45 \\
\hline $\begin{array}{l}\text { Hospitalisé ou hos- } \\
\text { pitalisée depuis un } \\
\text { an ou moins. } \\
C^{2}{ }^{2} 0,008\end{array}$ & 0,42 & $\begin{array}{l}\text { Perception d'une } \\
\text { santé déficiente ou } \\
\text { mauvaise. } \\
\text { Chi }^{2} 0,001 \\
\text { Souffre de solitude. } \\
\text { Chi }^{2} \text { non sign. }\end{array}$ & 0,41 & $\begin{array}{l}\text { Ne pouvant comp- } \\
\text { ter sur l'aide d'en- } \\
\text { fants pour l'en- } \\
\text { tretien ménager. } \\
\text { Chi' }^{2} \text { non sign. }\end{array}$ & 0,40 \\
\hline
\end{tabular}

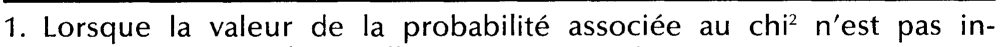
diquée, cela signifie qu'elle est supérieure à 0,05 .

du soutien social est manifestement pertinente dans l'explication de la relation personnes âgées-services du réseau des affaires sociales.

Deux autres dimensions méritent aussi d'être signalées : la réalité du groupe des 75 ans et plus et l'influence de la cohabitation 
enfants-personnes âgées. Lorsqu'on fait référence aux personnes âgées, on occulte souvent le fait que, sur le plan de l'âge, elles ne forment pas un bloc monolithique, qu'il y aurait nécessité, à tout le moins, de distinguer entre le groupe des 65-74 ans et celui des 75 ans et plus. Les résultats de notre étude vont dans cette direction; en cela, ils font écho à d'autres recherches qui ont évoqué le piège de l'homogénéisation des personnes âgées et qui ont mis en perspective des réalités différentes selon les groupes d'âge (Massé et Brault, 1984; Mathews, 1988; Roy, 1989). Notamment, notre étude a révélé que les personnes âgées de 75 ans et plus étaient davantage dépendantes du réseau des services publics, qu'elles désiraient deux fois plus que les autres être hébergées en institution, qu'elles étaient davantage en retrait de la vie sociale et communautaire; qu'elles vivaient davantage seules, sans conjoint, sans revenus suffisants, en proie à une solitude plus marquée. Or, dans ce contexte, la question du soutien social apparaît plus déterminante chez le groupe des 75 ans et plus, d'autant plus que, parmi les acteurs du soutien social, on retrouve en plus grand nombre des personnes âgées de 65 à 74 ans qui apportent aide et soins à leurs aînés. C'est l'expression d'une solidarité horizontale montante.

Une autre constatation de la recherche : la cohabitation des personnes âgées avec leurs enfants n'aurait pas à proprement parler un effet immunitaire sur le recours aux services du réseau des affaires sociales chez les personnes âgées. Rien dans les résultats de l'étude ne laisse transparaître une quelconque tendance, dans un sens comme dans l'autre. La cohabitation serait "neutre ". Pourtant, les recherches tendent généralement à démontrer que la présence d'aide fournie par le milieu familial, dont les enfants, est associée à un risque moindre de recours à l'hébergement institutionnel (Trahan, 1989). Par ailleurs, l'étude de Morris et al. (1988) mettait en évidence le fait que les personnes âgées demeurant avec un de leurs enfants présentaient un risque plus élevé d'être hébergées en institution. Shapiro et Tate (1985) avaient pour leur part observé que les personnes âgées ayant des contacts plus fréquents avec des membres de leur famille étaient davantage à risque d'hébergement; les auteurs expliquaient ces résultats par l'épuisement potentiel des familles en cause quant à l'aide à fournir aux parents âgés. Pour comparer nos résultats avec ces études, il nous aurait fallu probablement distinguer la question de l'aide des enfants avec celle de la cohabitation; également, le profil des personnes âgées cohabitant avec leurs enfants reproduit peut-être l'image de personnes moins autonomes sur le plan fonctionnel, donc plus à risque sur le plan de l'institutionnalisation. Notre étude n'a pas 
poussé plus avant cette investigation. Abordons maintenant les conclusions qui nous sont révélées par les entrevues semi-dirigées.

\section{Le volet qualitatif de l'étude}

Dans le volet qualitatif de la recherche, nous avons tenté de pousser plus loin encore nos connaissances sur ce qui, dans l'environnement d'une personne âgée, pouvait ou non favoriser son orientation vers les services institutionnels. Dans cette perspective, deux aspects du soutien social ont retenu notre attention : 1) les stratégies développées par les personnes âgées pour faire face à leurs limitations fonctionnelles; 2 ) la nature des rapports que les aînés et les aidants principaux entretiennent avec la communauté (voisins, groupes, associations, etc.). Ces deux thèmes qui nous renvoient de façon explicite à l'influence du soutien social ont été abordés à partir du concept central de "stratégies » qui renvoie, tel qu'il est défini par Sheriff et Lopez (1985), aux modes et aux manières que les personnes âgées ont d'organiser leurs relations, le système d'échange, les différentes actions et leur propre comportement en vue de faire face à leurs problèmes ${ }^{2}$. Autrement dit, il s'agissait pour nous de découvrir qui aide la personne âgée dans sa vie quotidienne. Quel type d'aide reçoit-elle ? À quel rythme? etc. Ce sont les résultats sommaires de cette exploration qui seront rapportés dans la présente section. Soulignons par ailleurs les limites de notre examen, puisqu'il s'applique à un échantillon réduit de 20 personnes âgées en perte d'autonomie et de 10 aidants naturels les soutenant.

\section{Les stratégies développées par les personnes âgées pour faire face à leurs limitations fonctionnelles}

L'étude des stratégies développées par les personnes âgées nous permet de mettre en lumière le soutien qui est disponible dans leur milieu naturel. Nous présentons ici les résultats par séquence, selon le type de ménage de la personne âgée.

Les personnes âgées qui cohabitent avec leur conjoint ou leur conjointe et un enfant (ou plus)

II ressort de notre étude que plus une personne âgée partage son univers immédiat avec un grand nombre de personnes significatives (enfants et conjoint ou conjointe, etc.) et plus elle évalue positivement sa situation. Elle estime aussi que la réponse apportée 
à ses besoins est à la fois suffisante et satisfaisante. La multiplicité des sources d'aide naturelle est donc associée, ici, au bien-être des personnes âgées.

Les ménages caractérisés par la corésidence enfant-parent âgé et conjoint ou conjointe de celui-ci $(\mathrm{N}=3)$ tendent généralement à s'autosuffire. Ils n'ont que très peu recours aux services extérieurs (dont les services formels de l'État) si l'on excepte, toutefois, le cas d'un aîné dans notre échantillon dont la condition médicale nécessitait des soins infirmiers spécialisés.

Les entrevues, malheureusement, ne nous ont pas permis d'établir la répartition des tâches et le degré d'engagement respectif entre le conjoint et le ou les enfants qui participent au soutien apporté à la personne âgée. Certains auteurs (Horowitz, 1985 cité dans Bolduc et Garant, 1990) ont toutefois observé que, plus que toute autre catégorie d'aidants, le conjoint ou la conjointe consacrait davantage de temps à son rôle de soutien ${ }^{3}$.

Dans l'ensemble, il est donc juste de dire qu'en dehors de l'unité domestique, très peu d'acteurs sociaux interviennent pour soutenir la personne âgée vivant dans un tel contexte. Dans une étude récente, Lesemann et Chaume (1989) sont également parvenus à cette conclusion ${ }^{4}$. Également, l'autonomie du ménage la préserverait d'un recours plus fréquent aux services du réseau de la santé.

\section{Les personnes âgées corésidant avec un enfant}

Les personnes âgées qui sont sans conjoint mais qui vivent avec une fille ou un fils agissant auprès d'elles à titre de personnesoutien se disent, sans exception, satisfaites de leur situation $(N=9)$. La plupart $(n=7)$ affirment en effet se sentir très bien et n'avoir que très peu de problèmes dans leur fonctionnement quotidien. De façon unanime, elles ont aussi reconnu que leur enfantsoutien est « bon » et s'acquitte très bien des responsabilités qui lui incombent.

Pour ces personnes âgées, aucun problème tangible n'a donc été identifié. Du côté de la fille ou du fils qui assume le rôle d'aidant principal $(N=7 / 10)$, la situation de corésidence se pose toutefois autrement. La plupart d'entre eux $(n=6)$ incriminent en effet, et plus vivement encore que tous les autres types d'aidants ou d'aidantes, l'isolement très important dans lequel ils se retrouvent en ce qui a trait à la prise en charge du parent âgé. Dans les faits, les responsabilités leur reviennent de façon quasi exclusive et très peu 
d'acteurs, hormis eux, se révèlent actifs dans l'aide et le soutien apportés à la personne âgée. Les dimensions les plus névralgiques de leur rôle seraient liées à l'impossibilité de se divertir ainsi qu'à la surcharge qu'impose le travail domestique. Dans un tel contexte, il $n^{\prime}$ est pas imprudent de dire que les moyens qui font défaut sont ici davantage ceux de la personne-soutien que ceux de la personne âgée.

Il est important de souligner, par ailleurs, qu'en dépit du manque d'engagement soutenu de la famille, la participation ponctuelle de certains membres reste néanmoins manifeste. Ils interviennent pour des petits services, tels que "faire la popote", " déblayer l'entrée ", " faire des réparations sur la maison », " faire des commissions ", etc. À quelques reprises, on a aussi signalé la participation d'un frère ou d'une sœur pour les déplacements.

La difficulté qu'on éprouve à cerner l'étendue des tâches qui sont accomplies par la fille ou le fils qui habite avec un parent âgé est une autre caractéristique de ce type de corésidence. Les tâches accomplies sont en fait aussi diversifiées qu'elles varient d'un aidant à l'autre; elles vont des soins corporels à la préparation des repas, du soutien physique dans la pratique de certaines activités jusqu'aux divertissements et aux loisirs.

On peut donc dire, en résumé, que le fait de vivre avec un de ses enfants est une situation que les aînés évaluent de façon tout à fait positive. Malheureusement, pour les personnes-soutiens, cette situation se vit beaucoup plus difficilement. On déplore en effet l'isolement vécu sur le plan de la prise en charge et la nonimplication des autres membres de la famille. La lourdeur des tâches ménagères et l'impossibilité de se divertir apparaissent comme la pierre angulaire de ce malaise. Aussi était-il prévisible qu'à la question « Est-ce qu'il y a certaines ressources ou catégories de ressources sur lesquelles vous aimeriez pouvoir compter davantage ? " la plupart des personnes-soutiens aient répondu par des services de répit et d'entretien domestique.

Dans le contexte de la corésidence parent âgé-enfant, il est important de rappeler que le fils ou la fille se révèle, à peu de chose près, être le seul acteur social engagé dans le processus d'aide. Ajoutons à cela que l'absence d'assistance des services de l'État auprès des enfants soutenant un parent âgé peut contribuer au retrait éventuel de l'aide des enfants et, conséquemment, à un recours accru aux services formels de l'État chez la personne âgée. De fait, l'isolement et l'insatisfaction ressentis par les enfants constituent un indice de désengagement potentiel. 


\section{Les personnes âgées qui vivent avec leur conjoint} ou leur conjointe

Les personnes âgées qui habitent avec leur conjoint ou leur conjointe $(\mathrm{N}=6)$ ont évalué positivement leur situation. II semble toutefois que cette dernière suscite beaucoup d'inquiétudes. On redoute en effet la maladie et la mort qui, à n'importe quel moment, pourrait venir faucher le compagnon ou la compagne de vie. Pour plusieurs, la peur de perdre la seule aide disponible s'ajoute aussi à cette tourmente.

Si l'on considère que la plupart des ménages composés du couple personne âgée-conjoint ou conjointe vivent très repliés sur eux-mêmes, il est évident que ces craintes s'avèrent fondées. Dans ce type de cohabitation, le ou la partenaire est en effet la principale source d'aide, exception faite, toutefois, des cas où le conjoint est lui aussi malade $(n=2)$. C'est lui ou elle qui prend en charge l'ensemble des besoins qui sont aussi disparates que diversifiés. Ainsi, comme c'était le cas pour les enfants personnes-soutiens, il est très difficile de définir et spécifier l'étendue des responsabilités qu'ils assument. Il apparaît plus approprié de dire que leur prise en charge s'ajuste continuellement selon la nature, l'ampleur et le moment où surgissent les besoins.

Les entrevues ont fait ressortir, par ailleurs, que les personnes âgées qui vivent avec leur conjoint ou leur conjointe bénéficient aussi, fréquemment, d'une aide ponctuelle de la part de leurs enfants pour différents travaux : réparations à la maison, peinture, coupe et entrée de bois, déneigement, couture, etc. La participation de ces derniers a aussi été soulevée sur le plan des transports et des emplettes à faire. Pour ces deux services, l'aide des enfants serait toutefois plus soutenue que ponctuelle. Les neveux, les voisins et les amis, de façon beaucoup plus exceptionnelle cependant, mettraient également, à ce dernier palier, un peu de leur temps à contribution.

En résumé, on peut donc dire que les personnes âgées qui vivent et reçoivent de l'aide de leur conjoint ou conjointe ont eux aussi des stratégies très simples qui s'orchestrent autour de un, deux ou tout au plus trois acteurs sociaux. II est très rare que ces stratégies dépassent les frontières du camp familial. À l'avantscène, le compagnon ou la compagne de vie s'impose comme étant le principal pourvoyeur de l'aide qui est donnée. Exception faite des emplettes et du transport où les enfants ont habituellement une participation plus soutenue, ceux-ci ne s'impliquent que de façon ponctuelle pour tous les autres genres de services. Pour cette catégorie de personnes âgées, il apparaît que la présence du con- 
joint ou de la conjointe constitue un élément permettant d'éviter une dépendance accrue à l'égard des services formels.

Dans notre échantillon, nous n'avions que deux cas de personnes âgées vivant seules. Ces personnes présentaient deux traits distinctifs : elles ont généralement des stratégies plus diversifiées que les personnes âgées qui cohabitent avec d'autres membres de leur famille, c'est-à-dire des stratégies où interviennent un plus grand nombre de personnes-soutiens pour des services qui sont aussi mieux délimités. Elles ont par ailleurs des réticences importantes à demander de l'aide. Ces aspects n'autorisent aucune généralisation pour le groupe des personnes âgées vivant seules, étant donné notre échantillon très réduit. L'exploration du type de rapport que les aînés rencontrés en entrevue entretiennent avec la communauté nous permettra de compléter la description des stratégies utilisées par les personnes âgées pour faire face à leur manque d'autonomie.

\section{Les personnes âgées, les aidants principaux et le rapport à la communauté}

Les entrevues réalisées auprès des personnes âgées $(N=20)$ de la MRC de Pabok nous ont permis de constater que celles-ci n'ont à peu près pas de contacts soutenus et engagés avec leurs voisins, pas plus d'ailleurs qu'elles n'indiquent la présence d'amis véritables dans leur vie.

Cette absence de contacts avec l'entourage, que l'on pourrait associer dans un premier temps à l'âge des répondants et répondantes, se retrouve aussi chez les aidants principaux $(N=10)$. Pour ces derniers, les amis et les voisins ne sont donc pas des acteurs sociaux significatifs. Cette observation n'est pas sans rappeler, en fait, l'absence quasi systématique de ces derniers sur le plan de l'organisation de la vie quotidienne des personnes âgées. Dans l'ensemble, on peut donc dire que la famille reste non seulement la source quasi exclusive de soutien pour les personnes âgées mais aussi, pratiquement, leur seule référence sur le plan de la sociabilité. Il est intéressant de souligner par ailleurs que l'association qui est faite entre " intimité " et " famille » semble jouer un rôle important dans la retenue que plusieurs expriment à aller vers l'extérieur, "vers les autres" pour demander de l'aide ou tout simplement pour bavarder et se rencontrer.

L'absence de sociabilité qui est ici rapportée est une information qui diffère beaucoup de ce que nous avions fait ressortir dans le volet quantitatif. L'analyse des résultats obtenus à la suite du questionnaire nous mettait en effet en présence de gens qui affir- 
maient voir, dans une proportion de près de $45 \%$, des amis ou des voisins une fois par semaine et plus. II n'est pas impossible que la différence qui est ici signalée s'explique par le fait que les personnes âgées rencontrées en entrevue ont toutes été choisies parce qu'elles présentaient certains facteurs de risque les mettant en retrait de la communauté.

Il en est de même pour le niveau d'intégration à la communauté qui a été appréhendé, dans le volet quantitatif, à partir $\mathrm{d}^{\prime}$ indicateurs tels que l'appartenance et la participation à différents groupes sociaux et communautaires. En réalité, les entrevues nous mettent en présence d'une participation beaucoup plus faible que ce que le volet quantitatif avait déjà fait ressortir; les personnes âgées ne franchissent que très rarement les cloisons de l'espace familial pour assurer, à l'extérieur, une participation active dans différents groupes ou organismes sociaux et communautaires. Lorsqu'elles y participent, le plus souvent, ce sera sur une base ponctuelle. Ainsi, pas plus que les voisins et les amis ne s'impliquent véritablement dans le réseau d'aide qui s'organise autour des personnes âgées, pas plus ils ne se révèlent actifs quant à leur sociabilité. La participation des personnes âgées et des aidants principaux aux groupes communautaires et sociaux semble par ailleurs très limitée. Dans les faits, la plupart des aînés que nous avons rencontrés sont repliés sur leur famille immédiate. Cette dernière leur fournit non seulement l'aide dont ils ont besoin, mais constitue aussi leur seul réseau de sociabilité.

\section{Discussion}

L'objectif de cette étude était d'identifier les facteurs (en particulier les facteurs environnementaux) qui conditionnent l'orientation des aînés, hommes et femmes, vers les différents services du réseau public. Essentiellement, comme nous I'avons vu, les facteurs environnementaux associés au recours aux services publics chez les personnes âgées s'apparentent davantage au micro et au méso-système tels que définis par Bronfenbrenner ${ }^{5}$ et sont donc liés au soutien naturel et à l'environnement social de la personne âgée plutôt qu'à des déterminants de type "santé ". (Voir le tableau du volet quantitatif de la recherche.) Cette première constatation revêt une importance particulière si l'on considère que, dans le réseau des affaires sociales, les pratiques et les critères rattachés à l'admissibilité aux services (en particulier à I'hébergement institutionnel) sont généralement fondés sur des facteurs de type "santé ", par exemple sur le volume hebdoma- 
daire d'heures requises en soins infirmiers. Or, notre recherche a plutôt mis en évidence le fait que certains facteurs tels que la perception d'une mauvaise santé, ou des limitations à l'autonomie fonctionnelle, exerçaient une influence sur le recours aux services publics, mais que la portée de cette influence se révélait significativement moins importante que celle tenant aux facteurs environnementaux.

Un autre constat important qui ressort de notre étude est que les stratégies développées par les personnes âgées vivant en milieu naturel sur le territoire de la MRC de Pabok sont très limitées. En fait, elles ne reçoivent de soutien que d'un nombre très restreint d'acteurs pour ne pas dire que, le plus souvent, ce soutien provient d'une seule et même personne. Cette situation a pour effet de créer chez l'aidant principal, surtout lorsque celui-ci est l'enfant de la personne âgée, un essoufflement important et de nombreuses insatisfactions. Pour la personne âgée, i l y a aussi une contrepartie à cette situation en ce sens que tout ce qui risque d'affecter, d'atteindre ou de perturber l'aidant principal risque aussi d'avoir des retombées immédiates sur la prestation du soutien qui lui est apporté.

Dans l'ensemble, on peut donc dire que le soutien social dont bénéficiaient les personnes âgées rencontrées est à la fois circonscrit et précaire. Par conséquent, l'environnement dans lequel elles vivent présente, lui aussi, une certaine fragilité. Or, selon nos résultats, c'est justement le déséquilibre de ce dernier qui entraîne le recours aux services publics; il devient alors de plus en plus évident que les moyens pris pour contrer cette tendance devront nécessairement avoir pour effet de soutenir et de maintenir l'équilibre dans l'environnement social et physique des personnes âgées. Cet équilibre, tout porte à le croire, est intimement lié au maintien et au développement du soutien social disponible.

La responsabilité des communautés, même si elle est souhaitable, $n^{\prime}$ est pas une force sur laquelle on peut actuellement compter. $C^{\prime}$ est un constat qui ressort très clairement de notre étude. En fait, ce n'est pas la communauté mais la personne dans la communauté qui soutient et prend en charge les aînés. Pour contrebalancer ce cloisonnement des responsabilités, il sera nécessaire de briser I'isolement des aidants principaux et de mettre en place des mesures qui permettront de stimuler le potentiel de prise en charge des communautés, sans pour autant reporter sur elle la responsabilité absolue des aînés.

D'autres actions devront aussi être prises. L'une des plus importantes est sans aucun doute le développement de services de soutien et de répit pour les personnes-soutiens (gardiennage, lits 
de répit, services d'entretien domestique, popotes roulantes, etc.). Nous croyons qu'il est en effet nécessaire que le réseau d'aide formelle augmente son assistance et joue, dans le futur, un rôle de plus en plus important à ce chapitre. II serait souhaitable que cette assistance emprunte toutefois de nouvelles formes, car les services de soutien et d'aide qui sont actuellement disponibles, en raison, entre autres, de leur faible intensité, ne répondent pas vraiment aux besoins qui ont été exprimés par les aidants naturels rencontrés à l'intérieur de notre recherche.

À l'instar de Jutras et Veilleux (1989), nous insistons aussi sur la pertinence d'offrir des services de soutien à domicile axés sur les tâches qui sont rattachées aux activités de la vie quotidienne (entretien ménager, hygiène corporelle, etc.). Jutras et Veilleux (1989) suggèrent également la constitution de groupes d'entraide, de soutien mutuel et $d$ 'interventions de soutien émotif pour répondre aux besoins d'assistance des aidants principaux et soulager, en partie, le fardeau qui est vécu. Le soutien à ce palier est très important. Il semble en effet que les cas d'abandon soient plutôt le fait d'aidants naturels à bout de ressources à la suite d'un engagement intensif et prolongé qu'un phénomène lié au désengagement des proches sur le plan des tendances sociales ou des valeurs (Bolduc et Garant, 1990). On comprend pour cette raison que les services ne doivent pas intervenir de façon résiduelle et une fois qu'ont été épuisés tous les recours du milieu naturel. L'insuffisance ou le retard à offrir des services de soutien risquent plus que toute autre chose de constituer un désincitatif à l'aide apportée par les proches et de contribuer ainsi à briser le tissu social plutôt qu'à le renforcer (Bolduc et Garant, 1990).

En résumé, et c'est peut-être là une des avenues les plus prometteuses sur le plan des services à définir, le soutien à la famille devra aussi se traduire par le développement de ressources intermédiaires. Outre le développement accru des services à domicile, on pense ici, par exemple, à des centres et des hôpitaux de jour, à des services d'hébergement temporaire à des fins de répit et à bien d'autres moyens qui offrent non seulement des soins spécialisés et appropriés aux personnes âgées, mais aussi le répit nécessaire aux personnes-soutiens pour qu'elles puissent poursuivre librement certaines activités personnelles sans compromettre la prise en charge qu'elles assument auprès de leur parent ou de leur proche âgé.

La réforme récemment présentée par le ministre de la Santé et des Services sociaux (MSSS, 1990) vient confirmer la tendance déjà exprimée par l'État de maintenir les personnes âgées dans leur milieu naturel et de mettre en œuvre des ressources favorisant leur 
maintien à domicile. Pour que cette orientation s'actualise favorablement, on devra asister à une redéfinition des rapports entre le milieu naturel et le milieu des services. Mais, surtout, mettre en place des conditions qui rendront favorable l'exercice du soutien aux personnes âgées, notamment en leur accordant une plus grande place dans les décisions qui les concernent (plans de services, milieu de vie...). Nous avons proposé ici quelques pistes de solutions qui, bien sûr, restent incomplètes. Néanmoins, elles ont l'avantage de mettre en lumière les articulations possibles entre le soutien social formel et informel. L'originalité de notre étude tient à l'intérêt que nous avons porté à l'examen de la relation personnes âgées et réseau de services formels; à cet égard, elle ouvre une réflexion tant sur des avenues relatives à de nouvelles définitions de services que sur des nouveaux champs de recherche à explorer.

\section{Notes}

1. Soulignons que ce projet de recherche s'inscrit dans les perspectives de recherche de l'équipe de recherche en gérontologie du Centre de recherche sur les services communautaires de l'Université Laval.

2. Voir, à ce sujet, Teresa Sheriff et Rosario Lopez-Tremblay, "Les stratégies des personnes âgées ", Service social, vol. 34, n 1, 1985, p. 77-89.

3. A. Horowitz, "Family Caregiving to the Frail Elderly », cité dans Louise Garant et Mario Bolduc, L'aide par les proches : mythes et réalités. Collection Études et analyses, MSSS, Direction planification et évaluation, Gouvernement du Québec, juin 1990, p. 41.

4. Frédéric Lesemann et Claire Chaume, Familles-providence : la part de l'État, Recherche sur le maintien à domicile, Groupe d'analyse des politiques sociales, Université de Montréal, Éditions Saint-Martin, 1989, $287 \mathrm{p}$.

5. Voir référence plus haut.

\section{Références bibliographiques}

BARRIAULT, C. (1984). Ressources : éléments de la problématique de santé dans la région 01. Département de santé communautaire, Hôtel-Dieu de Gaspé, $51 \mathrm{p}$.

BEAUDOIN, A. (1988). Services sociaux à l'enfance à la famille, aux personnes âgées et dans le domaine de la santé : l'état de la situation au Québec. Laboratoire de recherche, École de service social de I'Université Laval, $149 \mathrm{p}$.

BÉLAND, F. (1980). Méthodologie pour l'évaluation des programmes sociosanitaires : le cas des services à domicile pour personnes âgées. Laboratoire de recherches sociologiques, Université Laval. Cahier 15, 212 p. 
BÉLAND, F. (1982). Les principaux résultats de l'analyse des désirs d'hébergement de trois échantillons de personnes âgées du Québec. Québec : MAS, $35 \mathrm{p}$.

BÉLAND, F. (1984). "L'effet de la corésidence sur les désirs d'hébergement des personnes âgées ", Recherches socio-démographiques, vol. XXV, $n^{\circ} 2$ : 267-283.

BLANCHET, B. (1987). Évolution des ressources intermédiaires d'hébergement. Québec : Commission d'enquête sur les services de santé et les services sociaux. Dossier thématique, $80 \mathrm{p}$.

BOISVERT, R. et M. Martel. (1986). Les conditions de l'autonomie. Rapport d'enquête auprès des personnes âgées de 65 ans et plus vivant à domicile. Trois-Rivières : DSC de Sainte-Marie, $132 \mathrm{p}$.

BOLDUC, M. (1986). La politique et les programmes de service à domicile : constats et réflexions évaluatives. Québec : MSSS, 63 p.

BolduC, M. et L. Trahan (1988). Programme d'évaluation portant sur « le processus de réponse aux besoins de longue durée des personnes âgées ayant des limitations fonctionnelles ». (Devis général). Québec : Direction de l'évaluation, ministère de la Santé et des Services sociaux, $33 \mathrm{p}$.

BOLDUC, M. et L. Garant (1990). L'aide par les proches : mythes et réalités. Québec : Direction de l'évaluation, ministère de la Santé et des services sociaux, $157 \mathrm{p}$.

BRONFENBRENNER, U. (1979). The Ecology of Human Development : Experiments by Nature and Design. Cambridge, Mass. : Harvard University Press, $330 \mathrm{p}$.

CLÉMENT, M. (1989). La pauvreté dans Pabok : mythe ou réalité - Une étude exploratoire sur le vécu. Centre de recherche sur les services communautaires, Université Laval, $55 \mathrm{p}$.

ClÉMENT, M. (1990). Milieu naturel et milieu de services : l'interface recherchée. Étude exploratoire de la situation des personnes âgées dans la MRC de Pabok. Centre de recherche sur les services communautaires, Université Laval, 125 p.

Comité des soins de santé pour gens âgés de l'Association médicale canadienne. (1987). Les soins de santé pour gens âgés : les défis d'aujour$d^{\prime} h u i$, des solutions pour demain. Association médicale canadienne, $86 \mathrm{p}$.

Commission d'enquête sur les services de santé et les services sociaux. (1987). Dossier personnes âgées. Programme de consultation d'experts, $193 \mathrm{p}$.

CORIN, E. (1984). "Manières de vivre, manière de dire : réseau social et sociabilité quotidienne des personnes âgées au Québec », Question de culture. Québec : IQRC, 6 : 157-186.

CORIN, E. (1985). Le contexte social et culturel des problèmes socio-sanitaires en moyenne Côte-Nord: une perspective pour le développement des services communautaires. Rapport présenté à la Direction des services communautaires du Centre de santé Saint-Jean-Eudes à Havre-Saint-Pierre, $200 \mathrm{p}$. 
CORIN, E. et al. (1984). "Entre les services professionnels et les réseaux sociaux : les stratégies d'existence des personnes âgées ", Sociologie et Sociétés, vol. XVI, $\mathrm{n}^{\circ} 2: 89-104$.

CORIN, E. et al. (1984). Le fonctionnement des systèmes de support naturel des personnes âgées. Laboratoire de gérontologie sociale, Université Laval, III, $127 \mathrm{p}$.

CORIN, E. (1985). "La santé : Nouvelles conceptions, nouvelles images", dans Traité d'anthropologie médicale. Québec et Lyon : PUQ/IQRC/ Presses universitaires de Lyon, p. 45-73.

DELISLE, M.-A. (1987). La république du silence, solitude et vieillissement. Laboratoire de gérontologie sociale, Université Laval, 192 p.

DeLISLE, M.-A. (1988). "Que signifie la solitude pour les personnes âgées ? "Revue canadienne du vieillissement, vol. 7, $\mathrm{n}^{\circ} 4$ : 76-92.

DELISLE, M.-A. (1988). "La solitude des personnes âgées ", Santé et société, vol. $10, n^{\circ} 3: 41-44$.

DELISLE, M.-A. (1988). Les aspects psycho-sociaux de la fréquentation des urgences hospitalières par les personnes âgées. Exposé présenté dans le cadre des conférences organisées par l'École de psychologie de l'Université Laval, $11 \mathrm{p}$.

Département de santé communautaire du Centre hospitalier régional de la Beauce (1987). Le maintien à domicile des personnes en perte d'autonomie : le fardeau des aidants principaux, $46 \mathrm{p}$.

FAVROT, G. (1987). "Vieillir chez soi : un idéal pour tous, une contrainte pour les familles ", Prévenir: Les alternatives à l'hospitalisation. Cahiers d'étude et de réflexions édités par la Coopérative d'édition de la vie mutualiste, $\mathrm{n}^{\circ} 70: 41-56$.

FORTIN, A. et al. (1987). Histoire de famille et de réseaux : La sociabilité au Québec d'hier à demain. Montréal : Éditions Saint-Martin, 225 p.

GosSELIN, G. (1986). Étude exploratoire sur l'insécurité des adultes âgés de 65 ans et plus : ses composantes, ses fondements et ses rapports avec les demandes de relogement. DSC du CH Honoré-Mercier inc., 133 p.

GUBERMAN, N. et al. (1987). Amour, bain, comprimé ou l'Abc de la désinstitutionnalisation. Recherches synthèses et critiques publiées dans le cadre de la Commission Rochon, $\mathrm{n}^{\circ} 23: 263 \mathrm{p}$.

JUTRAS, S. et M. RENAUD (1987). Personnes âgées et aidants naturels, éléments pour une réflexion sur la prévention dans le plän d'ensemble "La santé pour tous ". Rapport global présenté à la Direction générale des services et de la promotion de la santé. Santé et Bien-être Canada, GRASP/SST.

JUTRAS, S. et F. VEILLEUX (1989). Des "partenaires » méconnus : les aidants des personnes âgées en perte d'autonomie. Groupe de recherche sur les aspects sociaux de la prévention (GRASP), Université de Montréal, $99 \mathrm{p.}$

LEPAGE, L. (1989). Le maintien à domicile des personnes en perte d'autonomie : le fardeau des aidants principaux. Centre de recherche sur les services communautaires, Université Laval, 109 p.

LeSEMANN, F. et C. Chaume (1989). Familles-providence, la part de l'État. Montréal : Éditions Saint-Martin, 287 p. 
MASSE, J.-C. et M.-M. BRAULT (1984). "Sociétés, vieillissement et stratification des âges », Santé et société, vol. XVI, n 2 : 3-14.

MATHEWS, G. (1988). Priorités de la recherche en matière de vieillissement. INRS-Urbanisation, $136 \mathrm{p}$.

MAuss, M. (1983). "Essai sur le don, forme et raison de l'échange dans les sociétés archaïques", dans Sociologie et anthropologie, France, Quadrize/PUF, p. 143-279.

Ministère de la Santé et des Services sociaux (1990). Une réforme axée sur le citoyen. Québec : MSSS, 91 p.

MORRIS, J.N. et al. (1988). "Inst.-risk II : An Approach to Forecasting Relative Rush of Future Institutional Placement », Health Services Research, 23(4) : 511-536.

PAQUET, M. (1089). "La famille et le maintien à domicile des personnes âgées ", Santé et société, vol. 11, n $4: 10-12$.

PAQUETTE, M. (1988). Le vécu des personnes soutien qui s'occupent d'une personne âgée en perte d'autonomie. DSC de Lanaudière.

Pitrou, A. (1983). "Des solidarités entre générations », Futuribles, p. 51-60.

Pitrou, A. (1986). Étapes du cycle de vie et vieillissement : modes d'approche et rapports entre générations. Aix-en-Provence : Laboratoire d'économie et de sociologie du travail, 247 p.

RoY, J. (1991). " Les personnes âgées vivant à domicile et le désir d'hébergement en institution ", Recherches sociographiques, vol. XXXI, $\mathrm{n}^{\circ} 2$, p. 227-239.

RoY, J. (1989). Étude exploratoire de la situation des personnes âgées vivant à domicile, dans le secteur des Chenaux, et leur désir d'hébergement institutionnel. Centre de recherche sur les services communautaires, Université Laval, $122 \mathrm{p}$.

RoY, J. (1988). "Les personnes âgées au Québec : Un avenir sombre ou prometteur? "Santé et société, vol. 10, nº $3: 36-40$.

SHERIFF, T. et R. TREMBLAY-LOPEZ (1985). "Les stratégies des personnes âgées ", Service social, vol. 34, n 1 : 77-89.

STRYCKMAN, J. et L. PARÉ-MORIN (1987). "Stratégies du maintien à domicile : l'apport des aidants naturels", Le Gérontophile, vol. 9, n 2 : 12-14.

THERRIEN, G. et P. BOUCHARD (1983). Les conditions de vie des gens âgés à domicile. Québec : ministère des Affaires sociales, $157 \mathrm{p}$.

TRAHAN, L. (1989). Les facteurs associés à l'orientation des personnes âgées dans les établissements d'hébergement : une revue de la littérature. Québec : ministère de la Santé et des Services sociaux, Direction de la planification et de l'évaluation, Collection Études et analyses, 5, $100 \mathrm{p}$.

ZOLA, I.K. (1981). "Culte de la santé et méfaits de la médicalisation », Médecine et société, les années 80 . Montréal : Éditions coopératives Albert Saint-Martin, p. 31-51. 